\title{
The Energy Momentum Spectrum of Quantum Fields
}

\author{
RICHARD V. KADISON* \\ University of Pennsylvania \\ Philadelphia, Pennsylvania, USA \\ Received October 1, 1966
}

\begin{abstract}
It is proved, assuming Einstein causality, that the energy-momentum spectrum of a quantum field cannot be bounded. More is known under special assumptions $[1,4]$. Our main concern is the method and general applicability of the result.
\end{abstract}

\section{Introduction}

The Haag-Araki formulation of local quantum field theory associates with open regions $\mathcal{O}$ of Minkowski space-time $R^{4}$ von Neumann algebras $\mathscr{R}(\mathcal{O})$ on a Hilbert space $\mathscr{H}$. The self-adjoint operators in $\mathscr{R}(\mathcal{O})$ correspond to the bounded observables of the field localized in the region $\mathcal{O}$ of spacetime. The dynamics and relativistic invariance of the field are expressed in terms of a (strongly-continuous) unitary representation $U$ of the Poincaré group $G$ on $\mathscr{H}$ in such a manner that $U(g) \mathscr{R}(\mathcal{O}) U(g)^{-1}$ $=\mathscr{R}(g(\mathcal{O})$ ), where $g(\mathcal{O})$ denotes the transform of the region $\mathcal{O}$ by the (inhomogeneous) Lorentz transformation $g$ of space-time. (This is covariance of $U$ and $\mathscr{R}$.) Further assumptions are made - among them:

$\left\{\mathscr{R}(\mathcal{O}): \mathcal{O}\right.$ open in $\left.R^{4}\right\}$ and $\left\{\mathscr{R}\left(\mathcal{O}_{s}\right):\left\{\mathcal{O}_{s}\right\}\right.$ an open covering of $\left.R^{4}\right\}$

both generate the same $C^{*}$-algebra $\mathfrak{A}$ (the quasi-local algebra of

the system).

$$
\begin{gathered}
\mathscr{R}\left(\mathcal{O}_{1}\right) \leqq \mathscr{R}\left(\mathcal{O}_{2}\right)^{\prime} \text { if } \mathcal{O}_{1} \text { and } \mathcal{O}_{2} \text { are space-like separated. } \\
\mathscr{R}\left(\mathcal{O}_{0}\right) \leqq \mathscr{R}(\mathcal{O}) \text { if } \mathcal{O}_{0} \leqq \mathcal{O} .
\end{gathered}
$$

According to the theory of unitary representations of locally compact abelian groups (generalization of Stone's theorem) [3: p. 147] the restriction of $U$ from $G$ to the 4-translation group (the additive group of $R^{4}$ ) gives rise to a projection-valued measure $E$ on the dual $\hat{R}^{4}$ of $R^{4}$, this dual being identified with energy-momentum space, such that $U(a)$ $=\int_{\hat{R}^{4}} \exp (i a \cdot p) d E(p)$. Stone's theorem tells us that each of the oneparameter unitary groups $t \rightarrow U(t a)$ has an infinitesimal generator $P_{a}$ which is a (not necessarily bounded) self-adjoint operator on $\mathscr{H}$. If $a$ is space-like $P_{a}$ is the momentum observable conjugate to translation in the direction $a$. If $a$ is a vector along the time axis, the generator $H$ is

* Research supported by ONR and NSF. 
identified with the total energy. The projection-valued measure $E$ on $\hat{R}^{4}$ is simply a synthesis of all the spectral resolutions of the momenta and energy observables. To speak of all the momenta and energy as having finite spectrum is to require that $E$ have support in a bounded region of $\hat{R}^{4}$ - equivalently, that $\|U(a)-I\| \rightarrow 0$ as $a \rightarrow 0$.

It is not the unitary operators $U(a)$ which are physically relevant, but rather the automorphisms $\alpha(a)$ defined by $\alpha(a)(A)=U(a) A U(-a)$ which they induce on the quasi-local algebra - so that, from the point of view of physical relevance, we should be concerned with a representation of $G$ by * automorphisms of $\mathfrak{A}$. There is, as yet, no theory associating a "spectrum" with such a representation: though it is clear that a restriction such as boundedness of the "spectrum" should be equivalent to: $\|\alpha(a)-\iota\| \rightarrow 0$ as $a \rightarrow 0$, where $\iota$ is the identity automorphism of $\mathfrak{A}$ and $\|\alpha(a)-\imath\|$ is the bound of $\alpha(a)-\iota$ as an operator on the normed space $\mathfrak{A}$. We say that $\alpha$ is a norm-continuous representation of $R^{4}$ by automorphisms of $\mathfrak{A}$, in this case. With this in mind, we make the:

Definition. A covariance system is a pair $\{\mathscr{R}, \alpha\}$ where $\mathscr{R}$ is a mapping which assigns a $C^{*}$-algebra $\mathscr{R}(\mathcal{O})$ to each bounded region $\mathcal{O}$ of $R^{4}$ satisfying (1), (2), and (3), and $\alpha$ is a representation of the additive group of $R^{4}$ by * automorphisms of $\mathfrak{A}$ satisfying $\alpha(a)(\mathscr{R}(\mathcal{O}))=\mathscr{R}(\mathcal{O}+a)$. We say that the system has bounded energy-momentum spectrum when $\alpha$ is norm-continuous.

\section{The Spectrum}

If $\mathscr{A}$ is a commutative $C^{*}$-algebra acting on a Hilbert space $\mathscr{H}$, it is easy to check that $\{\mathscr{R}, \alpha\}$, with $\mathscr{R}(\mathcal{O})=\mathfrak{A}$ for each bounded open $\mathcal{O}$ and $\alpha(a)=\iota$ for each $a$ in $R^{4}$, is a covariance system. We say that such a system is constant. Conversely, if $\mathscr{R}(\mathcal{O})=\mathfrak{A}$ for some bounded open $\mathcal{O}$, translating far enough in a space-like direction relative to $\mathcal{O}$, we see that $\mathfrak{A}$ is isomorphic with a subalgebra commuting with $\mathfrak{A}$. This subalgebra is in the center of $\mathfrak{A}$ and is abelian so that $\mathfrak{A}$ (isomorphic to it) is abelian.

Theorem. A covariance system with bounded energy-momentum spectrum is constant.

Proof. Since $t \rightarrow \alpha(t a)$ is a norm-continuous, one-parameter group of * automorphisms of $\mathfrak{A}$, there is a derivation $\delta$ of $\mathfrak{A}$ such that $\alpha(t a)$ $=\exp t \delta=\imath+t \delta+\frac{t^{2} \delta^{2}}{2}+\cdots$ (convergence in the norm topology on bounded operators on $\mathfrak{A})$ [2: Lemma 2]. Let $\mathcal{O}_{0}$ and $\mathcal{O}$ be the interiors of the spheres with center 0 , radii $r$ and $2 r$, respectively, in $R^{4}$. For each $a$ in $R^{4}$ and all sufficiently small $t, \mathcal{O}_{0}+t a \leqq \mathcal{O}$ so that $\alpha(t a)\left(\mathscr{R}\left(\mathscr{O}_{0}\right)\right) \leqq$ $\leqq \mathscr{R}(\mathcal{O})$, from (3). With $B$ in $\mathscr{R}(\mathcal{O})^{\prime}$ and $A$ in $\mathscr{R}\left(\mathcal{O}_{0}\right), B \alpha(t a)(A)-$ $-\alpha(t a)(A) B=0$ for small $t$. Thus $B A-A B+t(B \delta(A)-\delta(A) B)+$ $+\frac{1}{2} t^{2}\left(B \delta^{2}(A)-\delta^{2}(A) B\right)+\cdots=0$, for small $t$, so that $B \delta^{n}(A)-$ 
$-\delta^{n}(A) B=0$ for $n=0,1, \ldots$ Hence $B \alpha(t a)(A)-\alpha(t a)(A) B=0$ for all $t$ and each $a$ in $R^{4}$. It follows that $B$ commutes with $\mathscr{R}\left(\mathcal{O}_{0}+a\right)$ for all $a$; and, from (1), $B$ commutes with $\mathfrak{A}$. Thus, with $\mathcal{O}_{1}$ the interior of a sphere space-like separated from $\mathcal{O}, \mathscr{R}\left(\mathcal{O}_{1}\right) \leqq \mathscr{R}(\mathcal{O})^{\prime} \leqq \mathfrak{U}^{\prime}$. Since $\mathscr{R}\left(\mathcal{O}_{1}\right) \leqq$ $\leqq \mathfrak{A}, \mathscr{R}\left(\mathcal{O}_{1}\right)$ lies in the center of $\mathfrak{A}$. As each $\alpha(a)$ is an automorphism of $\mathfrak{A}, \alpha(a)\left(\mathscr{R}\left(\mathcal{O}_{1}\right)\right)\left(=\mathscr{R}\left(\mathcal{O}_{1}+a\right)\right)$ lies in the center of $\mathfrak{A}$; and, from (1), $\mathfrak{A}$ coincides with its center, that is, $\mathfrak{A}$ is abelian. From [2: Lemma 2] each $\alpha(a)$ arises from a unitary operator in $\mathfrak{A}^{\prime \prime}$; and, since $\mathfrak{H}^{\prime \prime}$ is abelian, $\alpha(a)=\iota$ for each $a$. For each open $\mathcal{O}_{2}, \alpha(a)\left(\mathscr{R}\left(\mathcal{O}_{2}\right)\right)=\mathscr{R}\left(\mathcal{O}_{2}\right)=\mathscr{R}\left(\mathcal{O}_{2}+a\right)$; and from $(1), \mathscr{R}\left(\mathcal{O}_{2}\right)=\mathfrak{A}$. Thus $\{\mathscr{R}, \alpha\}$ is constant.

Since the assumptions of quantum field theory rule out a commutative quasi-local algebra, we have:

Corollary. No quantum field has a bounded energy-momentum.

Of course the foregoing applies to covariance systems based on more general groups than $R^{4}$ (in particular, on $R^{n}$ ).

\section{References}

[1] Borchers, H.: Local rings and the connection of spin with statistics. Commun. Math. Phys. 1, 281-307 (1965).

[2] Kadison, R., and J. Ringrose: Derivations of operator algebras. Commun. Math. Phys. 4, 32-63 (1966).

[3] Loomrs, L.: An introduction to abstract harmonic analysis. New York: Van Nostrand 1953.

[4] Wightman, A.: Trieste lectures (1962). 